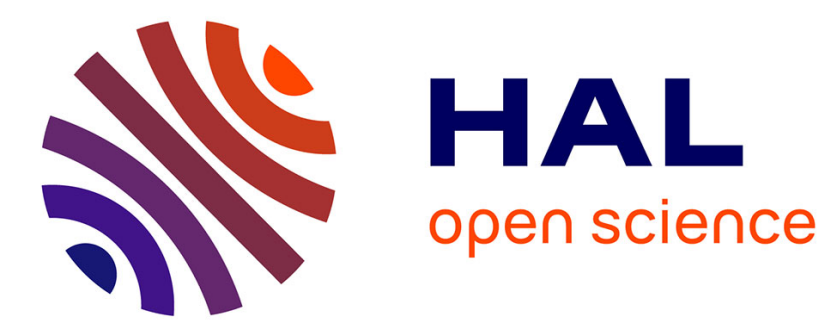

\title{
Resource Allocation and Admission Control in OFDMA-based Cloud-RAN
}

Mohammed Yazid Lyazidi, Nadjib Aitsaadi, Rami Langar

\section{To cite this version:}

Mohammed Yazid Lyazidi, Nadjib Aitsaadi, Rami Langar. Resource Allocation and Admission Control in OFDMA-based Cloud-RAN. GLOBECOM 2016 - 59th annual IEEE Global Communications Conference, IEEE ComSoc, Dec 2016, Washington D.C., United States. hal-01413769

\section{HAL Id: hal-01413769 \\ https://hal.science/hal-01413769}

Submitted on 11 Dec 2016

HAL is a multi-disciplinary open access archive for the deposit and dissemination of scientific research documents, whether they are published or not. The documents may come from teaching and research institutions in France or abroad, or from public or private research centers.
L'archive ouverte pluridisciplinaire HAL, est destinée au dépôt et à la diffusion de documents scientifiques de niveau recherche, publiés ou non, émanant des établissements d'enseignement et de recherche français ou étrangers, des laboratoires publics ou privés. 


\title{
Resource Allocation and Admission Control in OFDMA-based Cloud-RAN
}

\author{
Mohammed Yazid Lyazidi*, Nadjib Aitsaadi $^{\dagger}$ and Rami Langar ${ }^{\ddagger}$ \\ *Sorbonne Universities, UPMC Univ Paris 06, CNRS, LIP6 UMR 7606, 4 place Jussieu 75005 Paris, France. \\ ${ }^{\dagger}$ University Paris-Est, LIGM-CNRS UMR 8049, ESIEE Paris, 93162 Noisy-le-Grand, France. \\ ${ }_{\ddagger}^{\ddagger}$ University Paris-Est, LIGM-CNRS UMR 8049, UPEM, 77454 Marne-la-Valle, France. \\ Emails: yazid.lyazidi@lip6.fr, nadjib.aitsaadi@esiee.fr, rami.langar@u-pem.fr
}

\begin{abstract}
In this paper, we address the problem of downlink resource allocation and admission control for an Orthogonal Frequency Division Multiple Access (OFDMA)-based Cloud Radio Access Network (C-RAN). Specifically, we formulate the resource allocation and admission control for mobile users in C-RAN as an optimization problem, subject to constraints on mobile users data rate requirements, maximum transmission power and fronthaul links capacity. By dropping the non-linear constraint and reformulating the problem linearly using the framework of the well-known big- $M$ method, we propose a two-stage algorithm that can efficiently solve it. To satisfy the strict timing requirement of wireless communications in such a system, a time constraint was added to our algorithm. Numerical results demonstrate the good performance of our proposal in terms of number of accepted users and total transmission power, when compared with state-ofthe-art methods used for the control admission task in C-RAN.
\end{abstract}

Keywords: Cloud-RAN, LTE, Resource allocation, Quality of service, Admission control.

\section{INTRODUCTION}

With the high increase of mobile data traffic [1], building a new cost-effective Radio Access Network (RAN) that can address the deployments issues of traditional base stations and meet future 5G standards requirements has become business critical. C-RAN [1] has emerged as an innovative cloud architecture that can meet the expectations of current $4 \mathrm{G}$ and future $5 \mathrm{G}$ networks in terms of efficient utilization of baseband resources, flexibility and better interference management across multiple cells. Besides, C-RAN holds alluring benefits for network operators regarding CAPEX/OPEX cost savings and energy consumption reduction compared to traditional RAN structures [2].

In the C-RAN architecture, as illustrated in Fig. 1, BaseBand Units (BBUs) are located in a cloud or pool of virtual base stations. BBUs are based on high-programmable processors and virtualization technology that implement functions of the physical (PHY) and medium access (MAC) layers. The BBU pool is connected via a high-bandwidth low latency fronthaul network to Radio Remote Heads (RRHs) on the remote site. Fiber is the most common fronthaul solution used to convey the baseband data. In fact, Long Term Evolution's (LTE) imposes a strict timing delay for data processing inside the BBU pool. This imposes the fronthaul network to have a fixed capacity in terms of the digital baseband data that can be conveyed from the cloud to the RRHs in order to meet the strict LTE delay requirements [3].

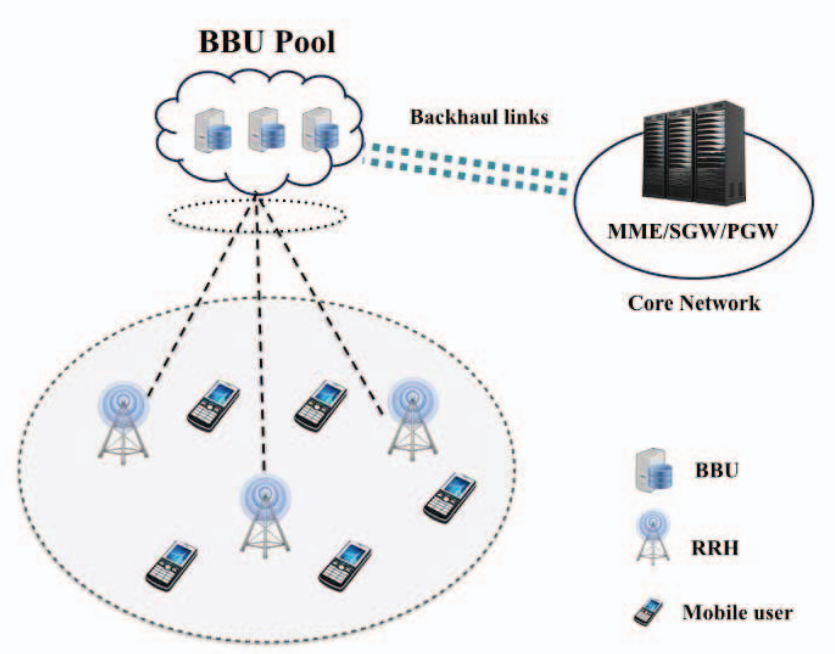

Fig. 1: Cloud Radio Access Network (C-RAN) Architecture

Since the fronthaul links separating the cloud to the radio site have finite capacity, a careful design must be performed regarding data transmission and fronthaul constraints. Additionally, C-RAN is challenged by the design of dynamic resource allocation and admission control algorithms that can cater to different users traffic profiles. Towards this end, we propose, in this paper, a joint resource allocation and admission control algorithm (RAAC) for downlink communications in OFDMAbased C-RAN, while considering mobile users Quality of Service (QoS) requirements in terms of achieved data rate, RRHs transmission power limitations, and fronthaul links capacity constraints. We formulate the problem as a Mixed Integer NonLinear Program (MINLP), which is NP-hard at first sight [4]. By dropping the non-linear constraint and reformulating the problem using the framework of the so-called big- $M$ method [5], the problem is rewritten as a Mixed Integer Linear Program (MILP) that can be efficiently solved using powerful MILP algorithms. We present then, a two-stage algorithm to address, firstly, the resource allocation problem. This is done by developing a fixed time branch-and-cut algorithm to solve the linear problem after a continuous relaxation of its integer variables. The second stage of our proposal addresses the admission control task, where users are accepted in our system based on their achieved data rate and QoS threshold. Simulations results exhibit the good performance of our proposal compared 
to state-of-the art approaches, in terms of number of supported users and total downlink transmitted power.

Our paper is organized as follows: Section II reviews the related work done in this context. Section III presents the assumptions and the mathematical formulation of our system model. In section IV, we present our algorithm design to solve the optimization problem based on the branch-and-cut and the big- $M$ linear reformulation. Performance evaluation of our proposal are discussed in section V, followed by Section VI that concludes the paper.

\section{RELATED WORK}

Resource allocation and admission control in C-RAN have recently received significant attention. Some proposals in this direction are [6]-[10]. In view of this, authors in [6] proposed low complexity algorithms to address these problems in two-tier C-RAN. Even though their proposal provides low complexity solutions for the addressed problems, their experimental results reveal their algorithms' limitation for only low QoS threshold regime. In fact, the percentage of total users admitted is less than $50 \%$ for a maximum QoS threshold of $11 \mathrm{~dB}$ and resource request of 3 sub-channels, which is quite underwhelming for current LTE cellular C-RAN systems.

The authors in [7] presented two Group Sparse Beamforming algorithms, to minimize the whole C-RAN power consumption. Their proposed algorithm successively switches off RRHs with minimum beamforming gain to minimize the whole power consumption. However, their study has been carried out separately, without considering the possible reconfigurations in the BBU pool. What is more, beamforming schemes may find limitations for computing-resource sharing, as highlighted by the authors in [8] who introduced a novel dynamic radio cooperation strategy for C-RAN to maximize the downlink weighted sumrate system utility, and which outperforms conventional RAN and beamforming schemes.

Authors in [9] proposed a downlink coordinated beamforming for a fronthaul constrained C-RAN. Their proposition aims at minimizing the total transmission power subject to QoS and maximum RRH power constraints. They presented a convex relaxation of their control admission task problem into a semi-definite program that is solved iteratively until convergence. However, works such as [6], [7] and [9] are based on snapshots models and do not measure the traffic time arrivals in their propositions. Such physical layer designs must include stochastic and time-varying constraints, so as to be applied in real-time context and consider the fluctuating traffic variation of mobile users.

In our previous work [10], we have proposed a novel approach for Physical Resource Blocks (PRBs) allocation and BBU-RRH assignment considering best effort mobile users. We presented a fixed time algorithm to determine at each user's arrival the optimal number of RRHs to be turned on as well as the number of needed BBUs to handle the whole traffic load. In this paper, we direct our focus to the admission control problem in C-RAN, where our aim consists in accepting as many users as possible in the system, while respecting their fixed QoS requirement in terms of data rate.

\section{System Model AND PROBlem Formulation}

In this section, we firstly discuss the physical layer assumptions and constraints for our considered downlink OFDMA C-RAN system model. The proposed RAAC design is then formulated as a MINLP problem based on these assumptions.

\section{A. System Model}

We consider a C-RAN system deployed in a large area consisting of $R$ RRHs (cells), denoted by the set $\mathcal{R}=\{r \mid 1 \leq$ $r \leq R\}$. We denote by $\mathcal{K}=\{k \mid 1 \leq k \leq K\}$, the set of $K$ available Physical Resource Blocks (PRBs) jointly assigned by the BBU pool to all $R$ RRHs. We consider $N$ Mobile Users (MU) from the set $\mathcal{U}=\{u \mid 1 \leq u \leq N\}$ located at different cells of the system. Each RRH $r$ has a unique antenna, which is serving all MUs present in its coverage zone. We consider that each MU $u$ requests from its serving RRH $r$ a number of resources, from a downlink channel bandwidth $B$, to meet his QoS target and run his mobile applications. Let $\alpha_{k, u}^{r}$ be the binary decision variable, which is equal to 1 if $\mathrm{PRB} k$ is allocated to MU $u$ from RRH $r$, or 0 otherwise. The transmitted power regarding this resource allocation is defined as follows:

$$
p_{k, u}^{r}= \begin{cases}p \in\left[p_{\min }, P_{\max }^{r}\right], & \text { if } \alpha_{k, u}^{r}=1, \\ 0, & \text { otherwise }\end{cases}
$$

where $p_{\min }$ is the minimum power that $\mathrm{MU} u$ equipment can receive and decode, and $P_{\max }^{r}$ is the maximum power allowed to be transmitted from an RRH $r$. We consider that a MU can only be attached to a single RRH, formally:

$$
\sum_{r \in \mathcal{R}} \alpha_{k, u}^{r} \leqslant 1
$$

We define the maximum transmission constraint for each RRH $r$ as:

$$
\sum_{u \in \mathcal{U}} \sum_{k=1}^{K} \alpha_{k, u}^{r} p_{k, u}^{r} \leq P_{\max }^{r},
$$

The SINR achieved by MU $u$ attached to RRH $r$ and on a given PRB $k$ can be expressed as:

$$
\gamma_{k, u}^{r}=\frac{p_{k, u}^{r} g_{k, u}^{r}}{\sum_{u^{\prime} \neq u} \sum_{r^{\prime} \neq r} p_{k, u^{\prime}}^{r^{\prime}} g_{k, u}^{r^{\prime}}+\sigma^{2}}
$$

where $g_{k, u}^{r}$ is the channel gain observed from RRH $r$ to MU $u$ on $\mathrm{PRB} k$ and $\sigma^{2}$ is the noise power. Based on the Shanon formula, we can express the achieved data rate of MU $u$ on all its received PRBs as:

$$
D_{u}=\sum_{r \in \mathcal{R}} \sum_{k=1}^{K} b_{u} \alpha_{k, u}^{r} B \log _{2}\left(1+\gamma_{k, u}^{r}\right)
$$

where $b_{u}, u \in \mathcal{U}$, represents MU $u$ 's priority in the system. For the sake of simplicity, we consider that $\forall u \in \mathcal{U}, b_{u}=1$. We suppose that the fronthaul network linking the BBU pool to the RRHs has a finite capacity in terms of baseband data that can be conveyed from the two sides. Besides, a part of the resource blocks usage is reserved for traffic signalization 
and control. Therefore, we impose a maximum traffic capacity $C_{\max }$ constraint for each RRH $r$ :

$$
C_{r}=\sum_{u \in \mathcal{U}} \sum_{k=1}^{K} \alpha_{k, u}^{r} \leqslant C_{\max },
$$

With the total traffic load observed at each RRH, we can estimate the number of needed BBUs in the cloud to manage the whole C-RAN traffic. Based on the formula proposed in [10], the number of needed BBUs $N_{B B U}$ can be expressed, by taking into account the overall network traffic load, as:

$$
N_{B B U}=\left\lceil\frac{\sum_{r \in \mathcal{R}} C_{r}}{K}\right\rceil
$$

where $\lceil$.$\rceil is the ceiling function. Note that other parameters$ can be included to estimate the total energy consumption in the cloud, such as the CPU, number of network interfacing cards and memory ressources needed to instantiate all $N_{B B U}$ in the virtual pool. The study of the resources and power consumption in the cloud are out of the scope of this paper.

\section{B. Resource Allocation and Admission Control problem formu- lation}

By considering the above constraints (2),(3),(6) and the individual MU's QoS target for a data rate threshold $D_{t h}$, our objective is to find the best PRB allocation to maximize the total throughput of all MUs in the system. Our optimization problem can be expressed as follows:

$$
\begin{array}{ll}
\max _{\alpha_{k, u}^{r}, p_{k, u}^{r}} & \sum_{u \in \mathcal{U}} \sum_{r \in \mathcal{R}} \sum_{k=1}^{K} \alpha_{k, u}^{r} \\
\text { subject to : } \quad & D_{u} \geq D_{t h}, \forall u \\
& \alpha_{k, u}^{r} p_{k, u}^{r} g_{k, u^{\prime}}^{r} \leq \Upsilon_{k, u^{\prime}}^{r^{\prime}}, \forall r \neq r^{\prime}, \forall u \neq u^{\prime} \\
& \sum_{u \in \mathcal{U}} \sum_{k=1}^{K} \alpha_{k, u}^{r} p_{k, u}^{r} \leq P_{\max }^{r}, \forall r \\
& \sum_{u \in \mathcal{U}} \sum_{k=1}^{K} \alpha_{k, u}^{r} \leqslant C_{\max }, \forall r \\
& \alpha_{k, u}^{r} p_{\min } \leq p_{k, u}^{r} \leq \alpha_{k, u}^{r} P_{\max }^{r}, \forall u, r, k \\
& \sum_{r \in \mathcal{R}} \alpha_{k, u}^{r} \leqslant 1, \forall u, k \\
& \sum_{u \in \mathcal{U}} \alpha_{k, u}^{r} \leqslant 1, \forall r, k \\
& \alpha_{k, u}^{r} \in\{0,1\}, \forall u, r, k
\end{array}
$$

Constraint (10) represents the interference constraint for neighboring MUs $u^{\prime}$ served by neighboring RRHs $r^{\prime}$ to $r$ on the same resource block $k$. Constraint (13) imposes $p_{k, u}^{r}=0$ if $\alpha_{k, u}^{r}=0$. Meanwhile, constraint (15) stresses the fact that two MUs served by the same RRH cannot share the same PRB. Finally, (16) means that $\alpha_{k, u}^{r}$ is binary. Our admission control task targets to select the largest set of MUs to be accepted in our system, so that all the above constraints can be satisfied. However, the formulated problem in (8) is MINLP whose solution is intractable, due to the non-linear logarithmic function in constraint (9) and the presence of both discrete and continuous variables. Besides, problem (8) is NP-hard [4].

In the next session, we present our proposed RAAC solution to linearize the problem and to solve it in a fixed amount of time by using the framework of the big- $M$ and branch-and-cut methods.

\section{Proposed RAAC Algorithm Design}

\section{A. Linear reformulation}

The non-convex product in constraint (10) between binary variable $\alpha_{k, u}^{r}$ and continuous variable $p_{k, u}^{r}$ can be linearized using the big-M modeling [5][10], since $p_{k, u}^{r}$ is explicitly bounded. The non-convex product $\alpha_{k, u}^{r} p_{k, u}^{r}$ can hence be replaced by a new variable $\beta_{k, u}^{r}$ and the following constraints will be added:

$$
\begin{array}{rll}
\forall r \neq r^{\prime}, \forall u \neq u^{\prime}, & \beta_{k, u}^{r} g_{k, u^{\prime}}^{r} & \leqslant \Upsilon_{k, u^{\prime}}^{r^{\prime}} \\
\alpha_{k, u}^{r} p_{\min } & \leqslant \beta_{k, u}^{r} & \leqslant \alpha_{k, u}^{r} P_{\max }^{r} \\
\left(1-\alpha_{k, u}^{r}\right) p_{\min } & \leqslant p_{k, u}^{r}-\beta_{k, u}^{r} & \leqslant\left(1-\alpha_{k, u}^{r}\right) P_{\max }^{r}
\end{array}
$$

On the other hand, the non-convexity in constraint (11) can be addressed by adding a big- $M$ constraint for imposing $p_{k, u}^{r}=0$, whenever $\alpha_{k, u}^{r}=0$ as in the following:

$$
\begin{array}{r}
m \alpha_{k, u}^{r} \leq p_{k, u}^{r} \leq M \alpha_{k, u}^{r}, \forall u, r, k \\
\sum_{u \in \mathcal{U}} \sum_{k=1}^{K} p_{k, u}^{r} \leq P_{\text {max }}^{r}, \forall r \\
p_{\text {min }} \leq p_{k, u}^{r} \leq P_{\text {max }}^{r}, \forall u, r, k
\end{array}
$$

where $m$ and $M$ are relatively small and big numbers, respectively. The new constraint (20) implies that if $\alpha_{k, u}^{r}$ is false (i.e., $\alpha_{k, u}^{r}=0$ ), $p_{k, u}^{r}$ is guaranteed to be equal to 0 , and is unconstrained otherwise. In this case, constraint (22) imposes the varying continuous variable to take values in the $\left[p_{\min }, P_{\max }^{r}\right]$ interval, which catches up with the definition in (1).

By omitting the non-convex data rate constraint (9), our resource management optimization problem can be expressed as:

$$
\begin{array}{ll}
\max _{\alpha_{k, u}^{r}, \beta_{k, u}^{r}, p_{k, u}^{r}} & \sum_{u \in \mathcal{U}} \sum_{r \in \mathcal{R}} \sum_{k=1}^{K} \alpha_{k, u}^{r} \\
\text { subject to } & (12)-(14)-(15)-(16), \\
& (17)-18)-(19)-(20)-(21)-(22)
\end{array}
$$

We divise then our optimization RAAC design into two stages. The first stage comprehends the resource management problem formalized in (23) without the data rate constraint. Based on the output solution of this first problem, we develop a fast admission control algorithm to find the set of accepted users in the C-RAN system while considering the dropped data rate constraint $(9)$.

\section{B. Proposed two-stage RAAC algorithm}

First of all, we solve the resource management problem formalized in (23) using a fixed time branch-and-cut algorithm. The branch-and-cut method is considered as a powerful algorithm that merges the advantages of both Gomory Cutting 
Planes and branch-and-bound schemes into one algorithm [11], which is much faster and reliable than the conventional branchand-bound. The algorithm is based on a linear relaxation of the integer variables into continuous ones, while adding Cutting Planes to enhance the problem's relaxation. This is essential to come closer to approximate integer solutions and speed up the convergence time. Considering our MILP problem formalized in (23), its variables are $\alpha_{k, u}^{r}, p_{k, u}^{r}$ and $\beta_{k, u}^{r}$. Since variable $\beta_{k, u}^{r}$ can be derived from the big- $M$ constraints (18) and (19), we focus our optimization on the "core" variables $\alpha_{k, u}^{r}$ and $p_{k, u}^{r}$. The pseudo-code of the proposed resource management problem is summarized in Algorithm 1. To further lessen the computation time of our algorithm, we add an upper bound limit $I_{\max }$ for the algorithm's maximum number of iterations. In fact, real-time resource allocation imposes a maximum ten seconds delay for mobile users connection to their serving RRHs [12]. If no connection has been established within this time, the RRHs (and consequently the BBU handling the user) will release the connection. Therefore, the number of iterations $I_{\max }$ should be scalable so as to not exceed the ten seconds period.

The pseudo-code of the control admission task is presented in Algorithm 2. After solving problem (23) and getting the output solutions for resource allocation and transmission power, we calculate the achieved data rate as in (5) for all MUs in $\mathcal{U}$ and check if their data rate target is achieved (i.e., $D_{u} \geq D_{t h}$ ). If so, all MUs are then accepted in the system; otherwise, we remove from $\mathcal{U}$ the $\mathrm{MU} u^{*}$ with the largest gap to its target QoS $D_{t h}$, and go back solving problem (23) with the updated set of MUs $\mathcal{U}=\mathcal{U} \backslash\left\{u^{*}\right\}$. We carry on the optimization until all MUs are accepted or $\mathcal{U}=\emptyset$. We may remark that Algorithm 2 is not optimal in terms of power consumption, however it finds practical use in real-time context where it returns a feasible solution of the set of accepted users in less than the aforementioned ten seconds, that are necessary before the connection's interruption.

\section{Performance Evaluation}

In this section, we evaluate the performance of our proposed RAAC algorithm and compare the benefits of our solution with respect to the semi-definite positive relaxation based algorithm (SDPRA) and Fast Greedy algorithm (FGA), which have been presented in [9] for admission control in C-RAN. Table I reports the simulation parameters that have been used for our experiments. We simulate a wireless LTE C-RAN environment consisting of 19 hexagonal-sized RRHs with radius $R_{\text {radius }}$. The distance between two nearest RRHs is $2 d$ and $R_{\text {radius }}=$ $2 d / \sqrt{3}, \forall r \in \mathcal{R}$. We consider 3 MUs uniformly distributed inside each cell, and the distance between a MU and its serving $\mathrm{RRH}$ is $d$ or $d / 2$. In what follows, we present the corresponding numerical results in terms of number of accepted MUs $N_{A M U}$, total transmission power in the C-RAN $T_{\text {power }}$ and number of BBUs $N_{B B U}$, while variating the QoS target $\gamma=2^{D_{t h} / B}-1$, and the fronthaul capacity $C_{\max }$. The results are obtained over 30 simulation instances for each scenario of $\gamma$ and $C_{m} a x$, with a confidence interval of $95 \%$. Repeated simulations helped us to fix the maximum number of iterations $I_{\max }$ to 500 for
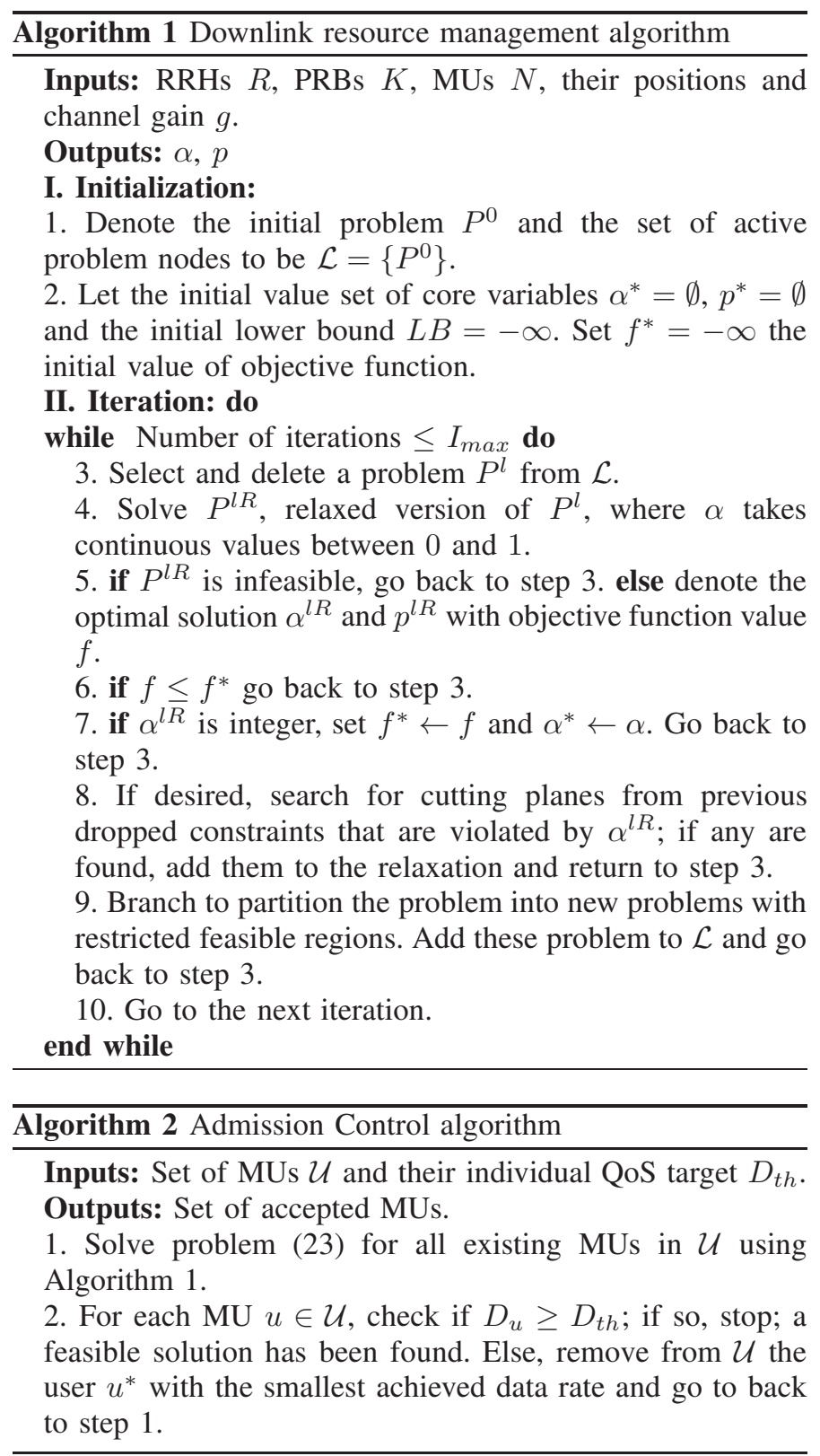

Algorithm 1, which allowed us to find close to optimal solutions while respecting the maximum time budget.

\section{A. Number of accepted MUs $\left(N_{A M U}\right)$}

Fig. 2 shows the evolution of the percentage of $N_{A M U}$ versus the QoS target $\gamma$ for the three studied schemes. As we can observe, the number of admitted MUs decreases with the increase of the target QoS level. Our proposed RAAC scheme results in increased number of supported users; up to $8.8 \%$ and $10.53 \%$ more admitted MUs compared to the SDPRA and FGA schemes, respectively. Then, we vary the fronthaul capacity $C_{\max }$ and study its impact on the percentage of accepted MUs. Fig. 3 illustrates the percentage of $N_{A M U}$ versus $\gamma$ for low $\left(C_{\max }=3\right)$ and high $\left(C_{\max }=9\right)$ fronthaul capacities. We remark that the number of supported MUs increases when the fronthaul network allows more baseband resources to be 
Table I: Simulation Parameters

\begin{tabular}{|c|c|}
\hline Parameters & Values \\
\hline Number of hexagonal cells & $R=19$ \\
\hline Number of MUs & $3 \mathrm{MUs} /$ cell $(N=57)$ \\
\hline Bandwidth & $B=5 \mathrm{MHz}$ \\
\hline Total number of PRBs & $K=24$ \\
\hline Path loss between RRH $r$ and MU $u$ & $43.8+36.8 \log _{10}\left(d_{r, u}\right)$ \\
\hline Carrier frequency & $2.5 \mathrm{GHz}$ \\
\hline Thermal noise & $-174 \mathrm{dBm} / \mathrm{Hz}$ \\
\hline Interference threshold & $1 \mathrm{~W}$ \\
\hline Fronthaul capacity $C_{\max }$ & $C_{\max } \in[3,24]($ default 6$)$ \\
\hline$P_{\max }^{r}$ & $10 W, \forall r \in \mathcal{R}$ \\
\hline$p_{\min }$ & $1 \mathrm{~mW}$ \\
\hline$d$ & $250 \mathrm{~m}$ \\
\hline$m(1 / M)$ & $10^{-6}$ \\
\hline
\end{tabular}

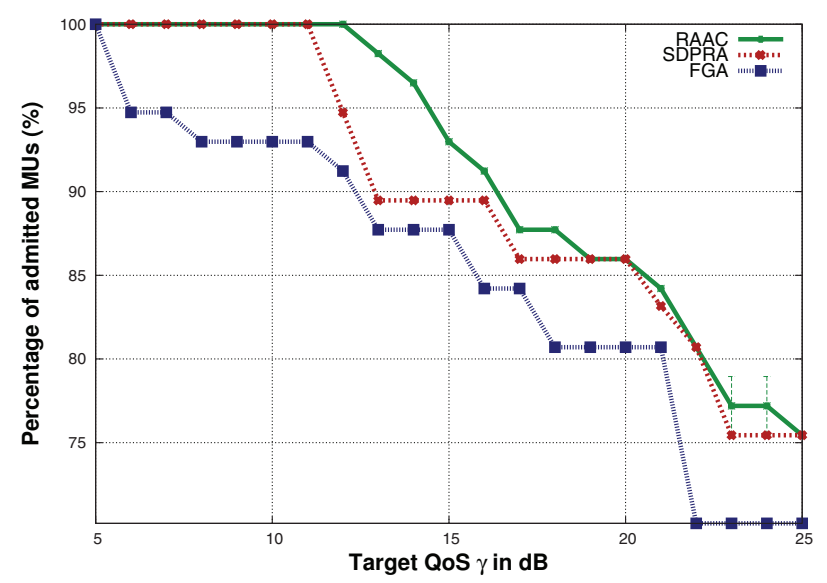

Fig. 2: Percentage of admitted MUs versus target QoS with fixed fronthaul capacity $C_{\max }=6$.

conveyed from the BBU cloud to the RRHs. As a result, this enables the C-RAN to allocate more PRBs to mobile users and satisfy their QoS requirement. Moreover, we notice that when RAAC and SDPRA achieve relatively the same results in high fronthaul capacity $C_{\max }=9$, the latter scheme remains outperformed by our proposal at low capacity $C_{\max }=3$, as can be observed in Fig. 2 and Fig. 3. This assesses the scalability of our proposal regarding increasing network traffic and fronthaul capacity.

\section{B. Total transmission power in C-RAN ( $\left.T_{\text {power }}\right)$}

We study the variations of the total transmission power $T_{\text {power }}$ for all RRHs versus the QoS target $\gamma$. As shown in Fig. 4, our method generates less transmission power than the SDPRA and FGA schemes for the same performance in number of admitted users. Furthermore, we notice that the total transmission power in both SDPRA and FGA present an unscaled behavior to the threshold regime $\gamma$, which implies that the power allocation is done irrespectively to MUs' QoS target. Our RAAC algorithm, on the other hand, shows that the total transmission power increases along with the growing QoS demand $\gamma$. We fix next the QoS target $\gamma=15 \mathrm{~dB}$ and study the total transmission power evolution versus the fronthaul capacity $C_{\max }$. Fig. 5 illustrates this evolution, where we observe that the total transmission power decreases as the fronthaul network capacity increases and allows more baseband resources to be

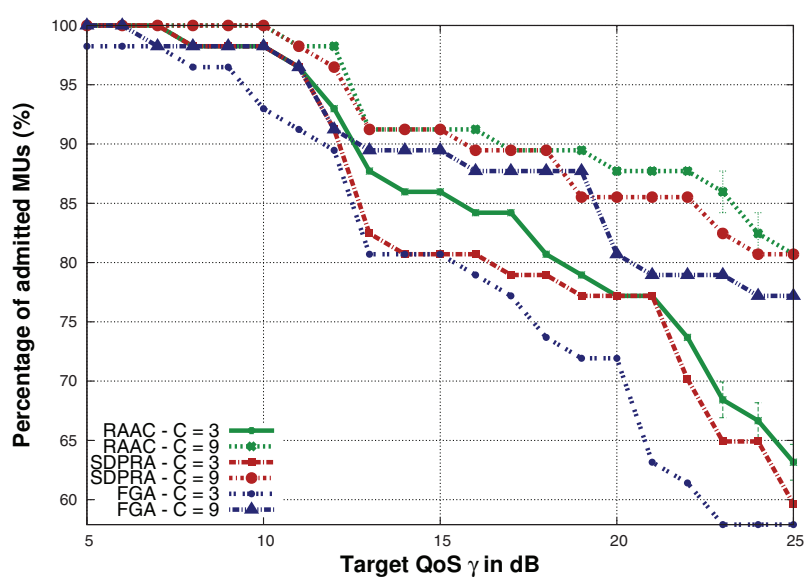

Fig. 3: Percentage of admitted MUs versus target QoS.

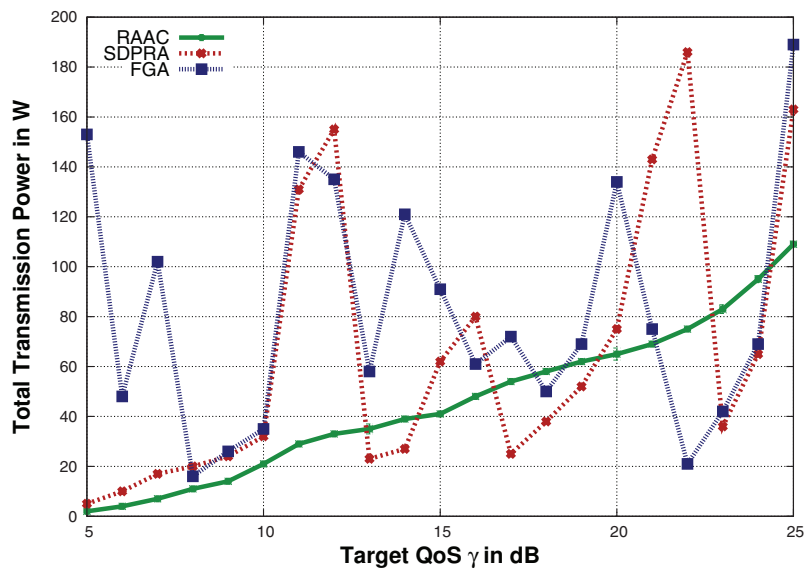

Fig. 4: Total transmission power versus target QoS with fixed fronthaul capacity $C_{\max }=6$.

transmitted. Besides, RAAC achieves more than $23 \%$ and $53 \%$ in power savings for large fronthaul capacity $\left(C_{\max } \geq 15\right)$ compared to SDPRA and FGA, respectively. This, once again, assesses the stability and good performance of our approach.

\section{Number of BBUs in the cloud $\left(N_{B B U}\right)$}

Fig. 6 presents the variation of number of needed BBUs for RAAC and traditional RAN scenario versus the data rate target, for fixed fronthaul capacity $C_{\max }=6$. Due to the one-one mapping in traditional RAN scenario, as many BBUs as deployed RRHs are needed to handle all 19 cells' traffic load. In centralized C-RAN, (provided the fronthaul links have sufficient capacity) one BBU can handle the traffic load of different RRHs, while meeting the existing users' data rate requirements. After the fronthaul limit is reached (for $D_{t h} \geq 35$ Mbps, as shown in Fig. 6), the number of BBUs is lessened, since more users will be rejected from the system because of their high QoS demand and/or their interference to other MUs. $N_{B B U}$ stabilizes then to 11-12 BBUs, which represents $42 \%$ savings compared to traditional RAN scenario. We present next the evolution of $N_{B B U}$ when the fronthaul capacity $C_{\max }$ varies and for fixed QoS target $\gamma=15 \mathrm{~dB}$. Fig. 7 depicts this 


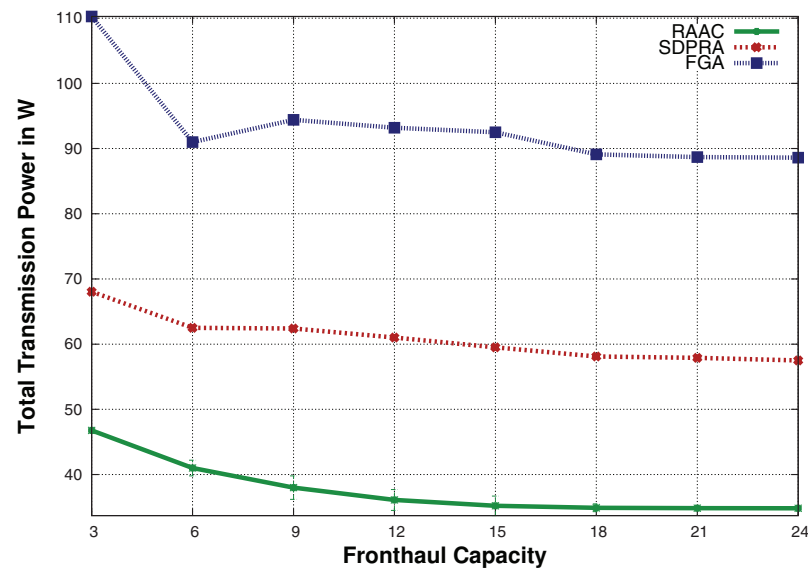

Fig. 5: Total transmission power versus fronthaul capacity $C_{\max }$ for $\gamma=15 \mathrm{~dB}$.

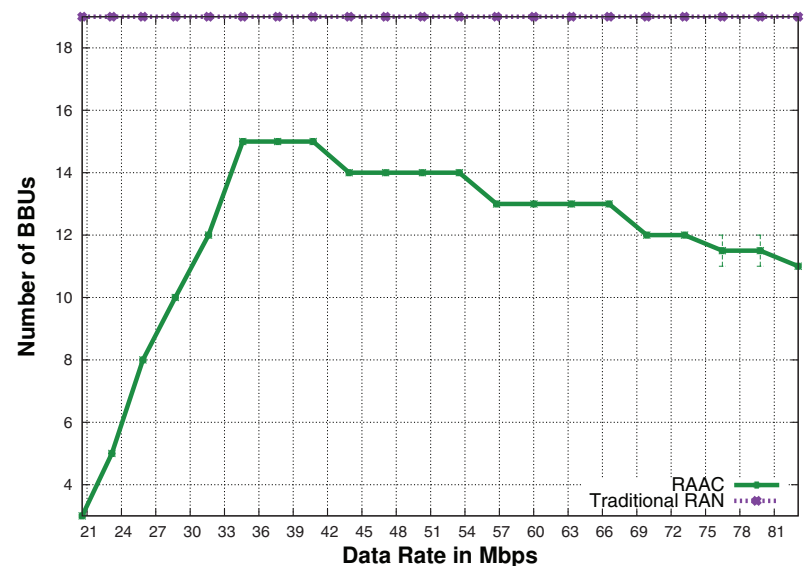

Fig. 6: Number of BBUs versus target data rate $D_{t h}$ for $C_{\max }=6$.

evolution for RAAC and the two state-of-the-art schemes. We remark that when the SDPRA and FGA schemes achieve less number of needed BBUs in the cloud for $C_{\max }<12$, they are outperformed by RAAC in terms of $N_{A M U}$ and $T_{\text {power }}$ as shown previously in Figs. 2 and 3, and Figs. 4 and 5, respectively. What is more, RAAC instantiates the same number of BBUs for $C_{\max } \geq 12$ as SDPRA and FGA, and for the same number of accepted users, which highlights the superiority of our proposal.

\section{CONCLUSION}

This work has focused on addressing jointly the resource allocation and admission control tasks in C-RAN, considering QoS requirements, interference and fronthaul network limitation constraints. We presented a fixed-time algorithm to solve the resource allocation problem and the admission control task. Numerical results have confirmed the performance of our proposed RAAC approach, which increases users' admission by $9 \%$ and $11 \%$, and saves $23 \%$ and $53 \%$ more C-RAN transmission power compared to the SDPRA and FGA schemes,

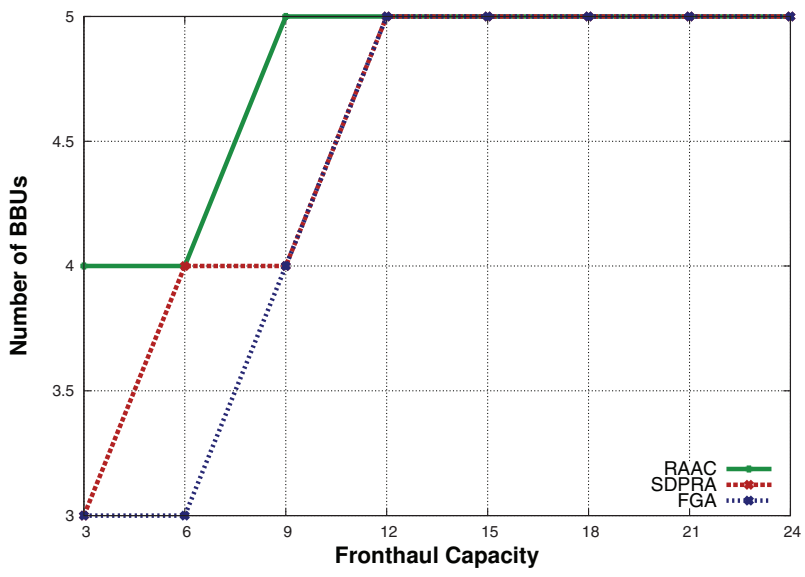

Fig. 7: Number of BBUs versus fronthaul capacity $C_{\max }$.

respectively. We also highlighted the benefits of our proposal regarding the number of BBUs' reduction in the cloud.

\section{ACKNOWLEDGMENT}

This work was partially supported by the ANR ABCD project (Grant no. ANR-13-INFR-0005-01) and the FUI ELASTIC Network project (Grant no. C16/0287).

\section{REFERENCES}

[1] T. Taleb, "Towards carrier cloud: Potential, challenges, and solutions," IEEE Wireless Communications Magazine, Vol. 21, No. 3, Jun. 2014. pp. 80-91, 2014.

[2] T. Taleb, M. Corici, C. Parada, A. Jamakovic, S. Ruffino, G. Karagiannis, and T. Magedanz, "EASE: EPC as a service to ease mobile core network," IEEE Network Magazine, Vol. 29, No. 2, pp.78 - 88, March 2015.

[3] N. J. Gomesa, P. Chancloub, P. Turnbullc, A. Mageec, and V. Jungnickeld "Fronthaul evolution: From CPRI to ethernet," Optical Fiber Technology - Next Generation Access Networks, vol. 26, Part A, pp. 50 - 58, 2015.

[4] A. Abdelnasser, E. Hossain, and D. I. Kim, "Clustering and resource allocation for dense femtocells in a two-tier cellular ofdma network,' IEEE Transactions on Wireless Communications, vol. 13, no. 3, pp. 16281641, March 2014.

[5] S. Leyffer, "Mixed integer nonlinear programming," Berlin, Germany: Springer-Verlag, 2012.

[6] A. Abdelnasser and E. Hossain, "Two-tier ofdma cellular cloud-ran: Joint resource allocation and admission control," in 2015 IEEE Global Communications Conference (GLOBECOM), Dec 2015, pp. 1-7.

[7] Y. Shi, J. Zhang, and K. Letaief, "Group sparse beamforming for green cloud-RAN," Wireless Communications, IEEE Transactions on, 2014.

[8] T. X. Tran and D. Pompili, "Dynamic radio cooperation for downlink cloud-rans with computing resource sharing," in Mobile Ad Hoc and Sensor Systems (MASS), 2015 IEEE 12th International Conference on, Oct 2015, pp. 118-126.

[9] V. Nguyen and L. B. Le, "Joint Coordinated Beamforming and Admission Control for Fronthaul Constrained Cloud-RAN," Globecom, 2014, Wireless Communications Symposium, 2014.

[10] M. Y. Lyazidi, N. Aitsaadi, and R. Langar, "Resource Allocation in Cloud-RAN with Real-Time RRH-BBU Assignment," IEEE International Conference on Communications, 2016. ICC '16, 2016.

[11] J. E. Mitchell, "Branch-and-Cut Algorithms for Combinatorial Optimization Problems," Mathematical Sciences Rensselaer Polytechnic Institute Troy, NY, USA, 1991.

[12] N. S. Networks, "LTE smartphones measurements," December 2011. [Online]. Available: http://networks.nokia.com/system/files/document/lte_ measurements_final.pdf 